(C) 2020 IEEE. Personal use of this material is permitted. Permission from IEEE must be obtained for all other uses, in any current or future media, including reprinting/republishing this material for advertising or promotional purposes, creating new collective works, for resale or redistribution to servers or lists, or reuse of any copyrighted component of this work in other works.

Full version of Barman, Nabajeet, Gc, Deepak and Martini, Maria (2020) Blockchain for video streaming : opportunities, challenges and open issues. Computer, 53, pp. 45-56. can be found at https://doi.ieeecomputersociety.org/10.1109/MC.2020.2989051 


\title{
Blockchain for Video Streaming: Opportunities, Challenges and Open Issues
}

\author{
Nabajeet Barman, Member, IEEE, Deepak G C, Member, IEEE, \\ and Maria G Martini, Senior Member, IEEE \\ Email: \{n.barman, d.gc, m.martini\}@kingston.ac.uk
}

\begin{abstract}
Blockchain, Quality of Experience (QoE), and Video Streaming have all received much attention from both academia and industry so far, although they have not been jointly addressed for prospective applications yet. While the industry has already adopted blockchain-based video streaming platforms, other stakeholders, e.g., academia, government, regulators, and service providers, could contribute more to develop protocols, technologies, and standards to help grow this niche technology and support its implementation in media streaming applications. This paper reviews the current technologies, industrial advancements, and critically identifies the current research activities and future research opportunities.
\end{abstract}

Index Terms-Video Streaming, Blockchain, Quality of Experience.

\section{INTRODUCTION}

W ITHIN the last decade, multimedia, in general, and video streaming, in particular, have radically changed the way we consume information and keep ourselves entertained. Video streaming services are being increasingly used, thanks to the recent Streaming Video-OnDemand (SVoD) services, such as Netflix, Hulu, and Amazon Prime Video, which are overtaking the traditional TV broadcast services in the UK [1]. The user expectation of any content, at any location, and at any time has resulted in the public acceptance and worldwide growth of such SVoD services. Furthermore, live gaming video streaming services, such as Twitch and YouTubeGaming, have also seen tremendous growth, with Twitch alone being the fourth highest peak internet traffic generator in the US, serving over 15 million active users daily with almost a million concurrent users [2]. It is important to note that the success of any service depends on user satisfaction, which can be characterized in terms of Quality of Experience (QoE) of the end-users. As a result, there was recently a focus shift from Quality of Service (QoS) based assessment of a service to QoE based assessment. Due to the proliferation of such streaming services and the new paradigm of user satisfaction measurement, both academia and industry have put in every effort towards the identification, design, and evaluation of QoE in such video streaming services.

Over the past few years, Distributed Ledger Technology (DLT), in general, and Blockchain, in particular, have gained increasing attention from various organizations worldwide, especially from financial services/banking, automotive, healthcare, insurance, public sectors, and education, amongst others. The digital ledger market for blockchain products and services is anticipated to reach $\$ 60.7$ billion

- Authors are with the School of Computer Science and Mathematics, Faculty of Science, Engineering and Computing, Kingston University, London, United Kingdom.

Manuscript received $x x x$; revised $\mathrm{XXX}$. in 2024, up from $\$ 708$ million in 2017 [3]. Blockchain itself is still a nascent technology, and much needs to be done to achieve its full potential so that it is essentially accepted by industry, government, and consumers. Blockchain-based video applications have already attracted the attention of the industry with streaming applications, such as Dlive and Livepeer, all already available in the market. The Gartner hype cycle [4] reports that the blockchain business for media applications, Blockchain in Media and Entertainment, is still at the very first stage of the "Innovation Trigger" phase and will take 5-10 years to reach the plateau of productivity as per current projections.

Due to the many prospective advantages provided by the blockchain technology (see Section 2.3), various research works have been carried out on exploring their application in various domains, from IoT [5] to addressing security issues in UAV networks [6] to video surveillance systems [7] to secure video storage [8]. However, there has been a little focus from academia on identifying the potential research questions and opportunities provided by the blockchain for video streaming applications. IBM executive report [9] discusses how blockchain has every potential to change the way how media content is currently transmitted, consumed, and paid for. Reports presented in [9] and [10] discuss how the video advertising industry, which is plagued by a lack of transparency, high middlemen charges and closed performance measurement metrics, can benefit from using the blockchain applications which provide a highly efficient and transparent advertisement platform at a reduced cost. A JPEG whitepaper [11] discusses the industrial needs, relevant use cases, and functionalities for media blockchain and the potential requirements of the ongoing standardisation activities within and outside JPEG. In [12] a distributed and tamper-proof novel watermarking multimedia blockchain framework for content processing and transaction is briefly discussed. Zhaofeng et al. in [13] presents a blockchain-based Digital Rights Management 
(DRM) system to help protect the content ownership rights while in [14], a blockchain-based platform ARCHANGEL for protecting video archives is presented. Ghimire et al. in [15] presents a novel video integrity mechanism based on blockchain for protecting from tampering. Detection of deepfake videos and images is a very challenging problem at present, for which blockchain-based solutions are being investigated in works such as in [16] and [17]. A detailed blockchain-based video delivery model using advanced network services chains has been proposed in [18]. The model provides a platform for collaboration among various providers using network service chains. Other works [19] [20] propose decentralized resource allocation for blockchain-based video streaming applications, with Mobile Edge Computing (MEC) to improve the video delivery to the end-user. Transcoding still remains a challenging task, especially for live streaming applications. Towards this direction, work in [21] presents a deep reinforcement learning based transcoder selection framework for blockchain-based video streaming solutions for more effective transcoding node selection.

Based on the above discussion it is clear that, while there have already been ongoing works on the potential use of blockchain technology for media and entertainment, an overview work exploring the current industrial and academic efforts, identifying various potential advantages and opportunities of blockchain with a focus on video streaming has been missing so far. Towards this end, in this paper, we present:

1) A discussion of the various opportunities provided by the use of blockchain technology for video streaming.

2) A review of the existing blockchain-based video streaming platforms.

3) A summary of the ongoing standardization activities on blockchain.

4) A conceptual framework for blockchain-based video streaming applications.

5) A discussion of the technical challenges and open issues.

\section{BACKGROUND}

\subsection{Video Streaming}

Video streaming platforms such as Netflix and YouTube use a centralized client-server architecture, where the multimedia content is delivered from a server to the client over the network. Such centralized systems face the problem of "last mile" delivery where, due to the low link capacity of the access network to the end-users, the QoS is significantly affected. The advent of new technologies, such as 5G and MEC, contributes to overcoming such limitations, with some new works exploring the potential of the blockchain technology, such as [19] and [20] discussed earlier. Still, many challenges remain due to the increasing bandwidth demand to meet the requirements of the changing entertainment landscape, involving the use of higher frame-rates and resolutions, i.e., $4 \mathrm{~K}$ and $8 \mathrm{~K}$, as well as newer video formats, e.g., High Dynamic Range (HDR), 3D, Light Field, and Point Clouds. A possible solution is the decentralized distribution of content through Peer to Peer (P2P) networks where the data is shared by participating nodes (clients). This sharing of bandwidth by the users of a network can help overcome the bottleneck "bandwidth" issue, resulting in a comparatively cost-effective, efficient, and faster network.

\subsection{Quality of Experience}

Over the past two decades, the industry mindset has shifted from a product-centric to a service-centric approach, where the users are at the center of the business model. Measuring or estimating the QoE is critical to understand the success of a service or technology. The adoption of a new technology by end-users is based on the level of user satisfaction, which for video streaming applications can be estimated using various QoE models and metrics. On the other hand, existing services can benefit from using the QoE models and metrics as it can be used to estimate and, as a result, improve the end-user satisfaction, which will ultimately result in increased viewing times and reduce user churn.

\subsection{Blockchain Technology - Features, Advantages and Shortcomings}

Blockchain is a digital list of records, i.e., blocks, which are linked together using cryptographic algorithms. It gained tremendous attention with the introduction and high popularity of Bitcoin, which is a cryptocurrency based on the blockchain technology. Its property of resistance to modification (immutability) makes it a highly prospective disruptive technology across a wide range of industries. Some of the significant advantages offered by blockchain include faster and secure transactions, transparency, costeffectiveness (due to the absence of middleman), traceability, automated actions using smart contracts, and cryptographically sealed protected data providing security and privacy. All these advantages come with their fair share of shortcomings, the most important of which is scalability. Due to the limited size of the block, the rate of mining is slow. Bigger block size results in slower propagation in the complex chain of blocks. Also, current blockchain technologies can handle a relatively low transactions rate, typically seven per second for Bitcoin and fifteen per second for Ethereum.

On an elementary level, blockchains can be broadly classified into three categories: Public (e.g., Bitcoin, and Ethereum) which are fully decentralized and available to all participants; Private (Hyperledger and R3 Corda) which are usually centralized and entities require permission from the blockchain owners, and Hybrid (also called Consortium blockchain, e.g., Dragonchain and Hyperledger Quilt) which combine the advantages of both the public (transparency and security) and private (privacy) blockchains.

Due to the increasing popularity of Blockchain, much like Platform as a Service (PaaS), Software as a Service (SaaS) and Infrastructure as a Service (IaaS), the concept of Blockchain as a Service (BaaS) is getting popular. Here, BaaS allows both companies and consumers to develop, test, and deploy secure blockchain apps with the required functionality, such as bandwidth management, resource allocation, and hosting requirements. Currently, BaaS is provided by major companies, such as IBM, Microsoft, and Oracle, and is increasingly being accepted by various industries because this allows them to focus on their product/service offering without having to worry about the underlying blockchain 

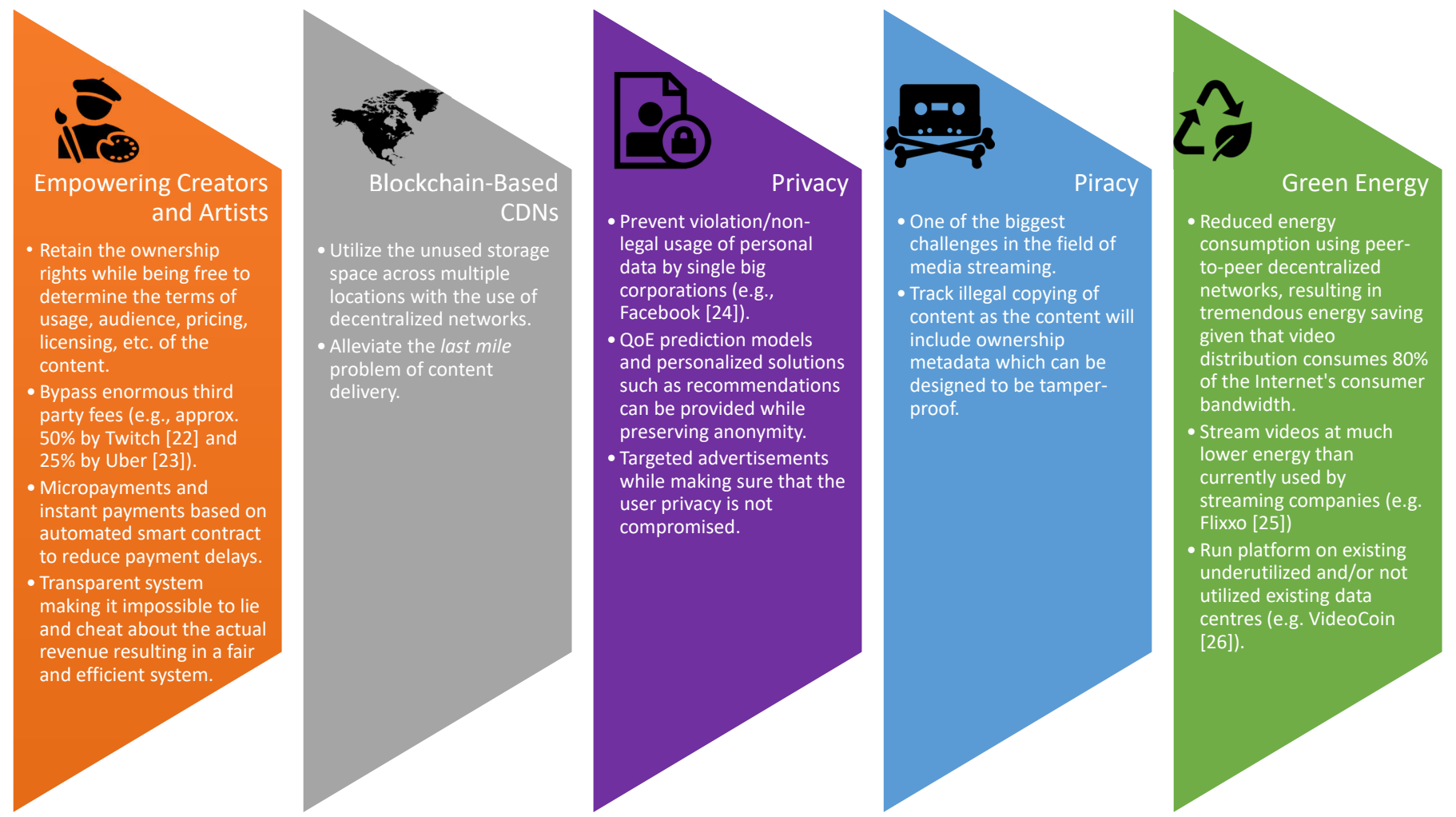

Fig. 1: Opportunities offered by blockchain technologies for video streaming application ( [22], [23], [24], [25], [26]).

network protocols and reference models. For a detailed overview of blockchain's fundamental concepts, see, e.g., [27] and for its possible impact on society, see, e.g., [28], and the references therein.

\section{Blockchain for Video Streaming}

Over the past few years, the world has seen a proliferation of smartphone users, which has dramatically changed the way we produce and consume digital content. People have taken up the role of content "producer", from recording/live streaming events, such as concerts and film festivals, to video blogs, e.g., travel, tutorials, product review, to live gaming video streaming. Limited by the shortcomings of the traditional platforms, which fail to reward the content creator and viewers adequately, we have seen in recent years the rise of platforms, such as Medium, Bonzo Me, and Tsu, which reward content producers and in some cases also the viewers, leading to a win-win environment for both content producer and viewers. One of the muchanticipated use cases for blockchain-based video streaming platforms is indeed in the domain of live streaming and gaming/eSports. Some of the major prospective advantages offered by blockchain-based video streaming applications include empowering creators and artists, blockchain-based CDNs, privacy and piracy protection, and energy saving, as presented in detail in Figure 1.

In order to shed light on the current market scenario, the state of technology adoption, and various challenges currently faced by the industry, we present in Table 1 a brief comparative review of six of the biggest companies providing blockchain-based video streaming solutions. We can observe that blockchain-based video streaming has already been introduced in the marketplace. In addition to these four companies, Play2Live, the world's first fullscale blockchain-based decentralized streaming platform using its own blockchain platform called Level Up Chain (LUC), needs mentioning. With a major focus on the eSports domain, Play2Live provided additional services such as betting and gambling. Due to regulatory challenges, the company ceased operations at the end of 2018, and its fate at the time of writing this article remains unknown. This indicates the need for international standards guided by technology and regulations so that the blockchain technology and its applications can grow further and get increasing acceptance worldwide. We present in Figure 2 the summary of the current ongoing standardization activities.

\section{A CONCEPTUAL BLOCKCHAIN-BASED APPLICATIONS \\ FRAMEWORK FOR
VIDEO STREAMING}

Based on the above discussion on the need for unifying the various ongoing works to achieve better, efficient, and interoperable systems, it is essential to design a general network architecture describing the multiple network elements and modules and how they interact with the new and existing systems. Towards this end, we present in Figure 3 a conceptual framework for blockchain-based video streaming applications considering the whole multimedia delivery chain from content production and transmission to content consumption. We follow an on-the-top modular approach based on the concept of BaaS, which offers the advantage that 
TABLE 1: Summary of the four blockchain-based video streaming companies.

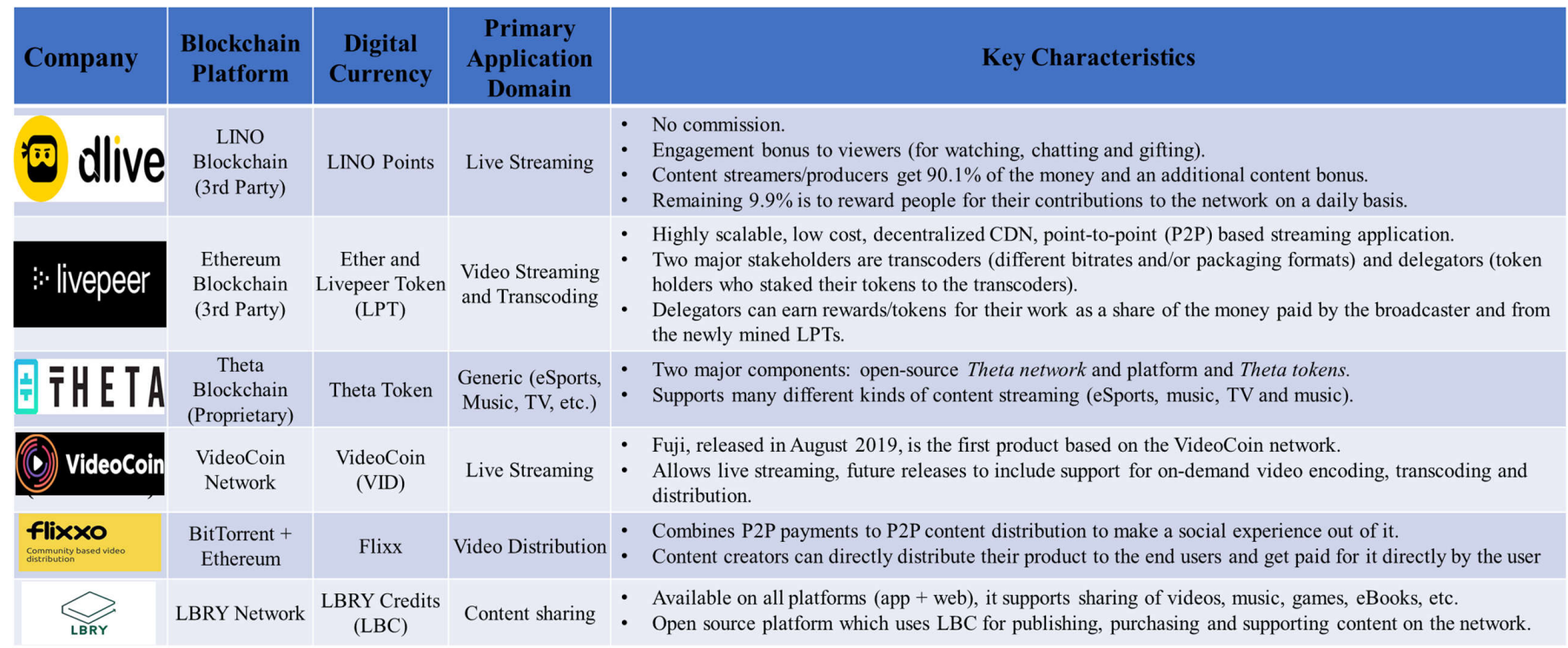
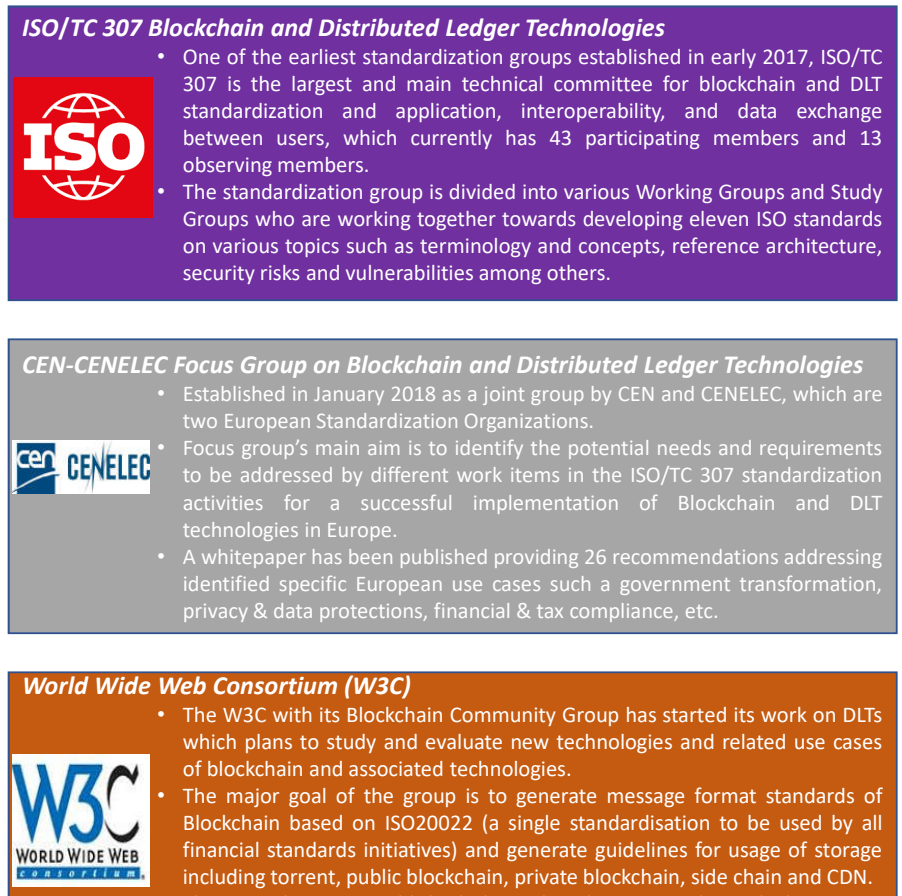

\section{- Consortium (WBC)}

The W3C with its Blockchain Community Group has started its work on DLTs which plans to study and evaluate new technologies and related use cases of blockchain and associated technologies.

The major goal of the group is to generate message format standards of Blockchain based on ISO20022 (a single standardisation to be used by all financial standards initiatives) and generate guidelines for usage of storage including torrent, public blockchain, private blockchain, side chain and CDN. The group has since published The Web Ledger Protocol 1.0 which presents a format and protocol for decentralized ledgers on the Web.
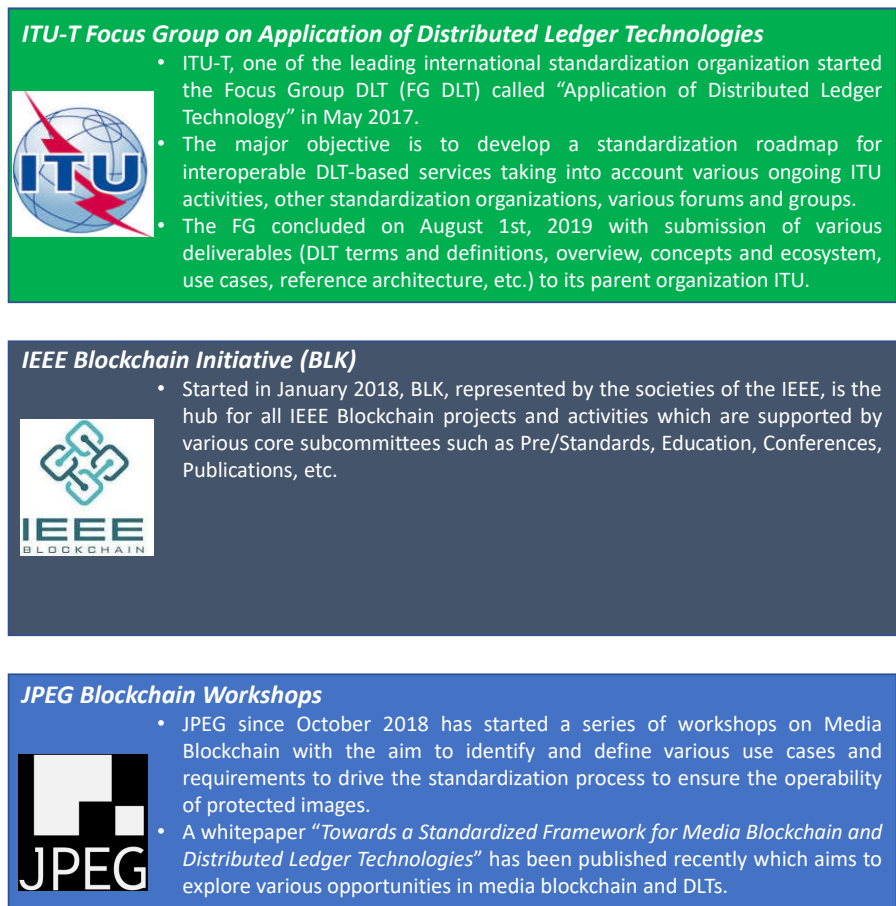
explore various opportunities in media blockchain and DLTs.

Fig. 2: Standardization activities.

depending on the streaming requirements, the blockchain application can be run either with newly designed networks or by integration into the existing applications or both. Due to the low-cost, open, and high scalability advantages offered by distributed, decentralized applications, there is already an effort towards the design of a decentralized web. For example, in the case of Content Delivery Network $(\mathrm{CDN})$, some existing centralized solutions such as Cloudflare, have already started offering "decentralized content gateway" via InterPlanetary File System (IPFS), which can be used by blockchain-based applications for decentralized file storage and access. Also, tools such as Hyperledger Quilt already offer interoperability between ledger systems for payments and transfer value across distributed and nondistributed ledgers.

However, there is still a lack of technological capabilities for supporting video streaming in a fully decentralized manner. Hence, the proposed conceptual framework is designed to offer a modular, highly scalable design, where the application developer has the flexibility to decide on the exact type of solution (centralized/decentralized, distributed) to be used at each stage of the multimedia delivery, depending 


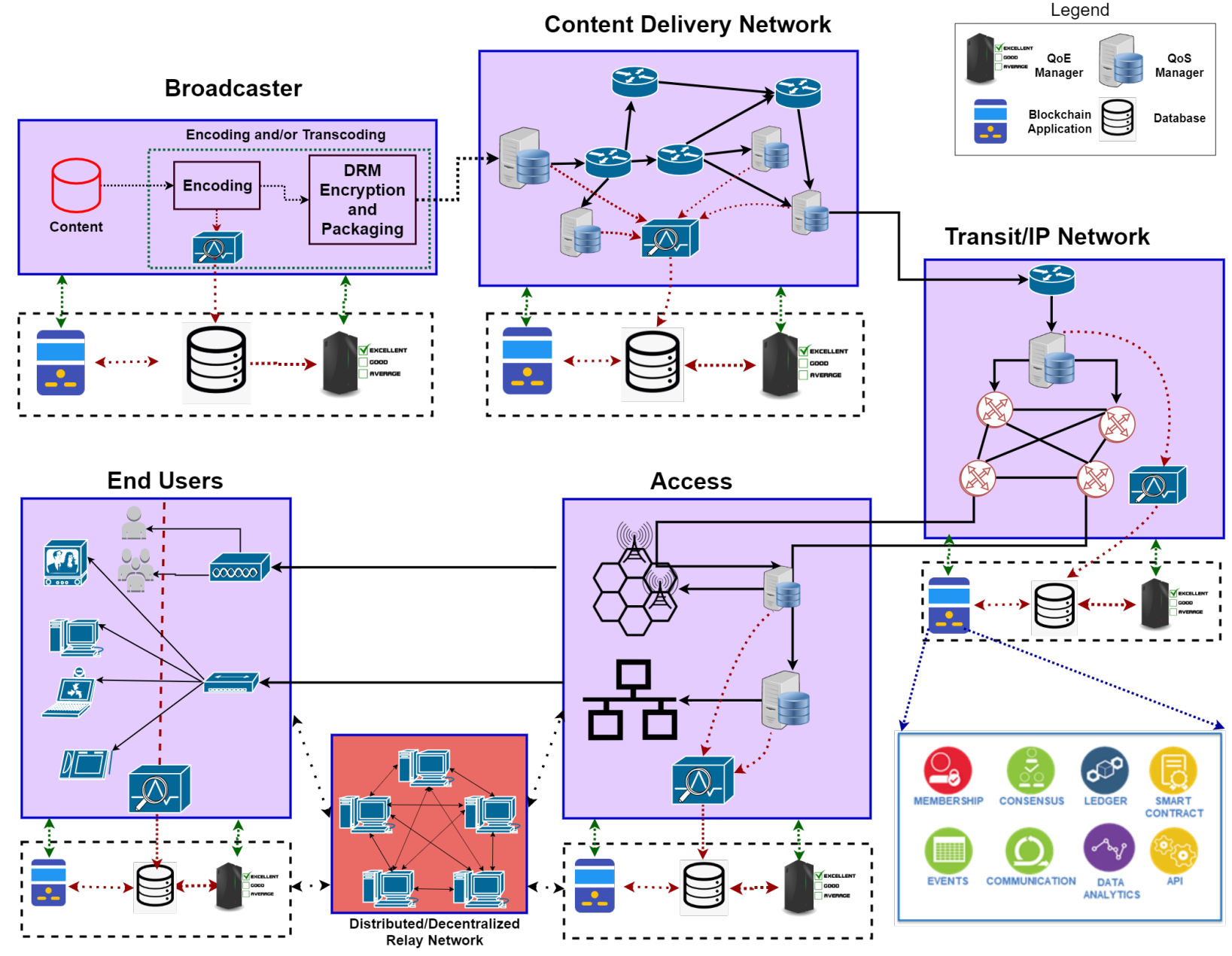

Fig. 3: Proposed conceptual framework for blockchain-based video streaming (Icons of components of blockchain application borrowed from IBM blockchain reference architecture template).

on the application requirements. We discuss next the three major roles one can select at the different stages of the multimedia delivery chain, as shown in Figure 3.

1) The broadcaster is an OTT service provider or a user who wants to deliver a video over the network to the viewers. To encode and/or transcode a video along with encryption and packaging, the broadcaster can use existing centralized solutions (e.g., cloud encoding solutions such as Amazon Web Services) or use decentralized transcoding solutions where various entities act as "transcoding" nodes to perform these tasks in return for payment for each task using digital currency (e.g., Bitcoin, Ether, etc.).

2) The CDN can be a centralized CDN solution (e.g., Akamai) or a decentralized CDN (such as VideoCoin and Theta Token), or a combination of both, as required.

3) The broadcaster can choose to use a distributed/decentralized network of "relay nodes" to help in the distribution of the video. Such relay nodes can help reduce network latency as well as provide high scalability. Music streaming applications such as Spotify use both the P2P network and centralized client-server architecture to deliver the fastest service possible to the end-users. Like transcoding nodes, the relay nodes get paid for the tasks performed using digital currencies. Finally, the video gets delivered to the end-users. The users pay the broadcaster after service delivery.

The proposed architecture contains four additional modules: Blockchain Application (BA), QoE Server, QoS Server, and Database. The BA is the core module implementing the blockchain framework. Depending on the application requirements, it can be a permissioned blockchain (e.g., hyperledger) or permissionless (e.g., ethereum) or a hybrid. Depending on the stage of the multimedia delivery and the type of underlying network (centralized/decentralized), the BA will consist of one or more of the following different functional groups (as shown in Figure 3):

1) Membership, e.g., participating nodes and their IDs, roles, rights, and permissions (such as hyperledger composer on top of Hyperledger fabric).

2) The ledger contains metadata such as timestamps, video content-related information (such as recording and publishing rights, ownership information), smart 
contract payment information (e.g., chaincode in hyperledger or ethereum).

3) Consensus algorithms, e.g., proof of work and proof of stake.

4) Smart contracts which can be executed automatically at the end of an event/completion of a transaction. For example, in the above proposed framework, automated payments from broadcaster to transcoding and/or relay nodes, payment from end-user to the broadcaster for using the service, etc. can be automatically triggered using pre-negotiated terms programmed into the smart contract.

5) The Application Programming Interfaces (APIs) to allow the BA to connect and interact with different network elements such as REST APIs.

6) The data analytics can provide an overall insight into the functioning of the various components such as QoE values, number of successful transactions, the overall number of nodes, etc., supplemented by various visualization and analysis tools.

7) The communication sub-module implements the connection protocols used and message type, for example, TCP/UDP based as in standard web applications or IPFS based decentralized protocol used for decentralized storage communication amongst various nodes.

8) The events sub-module detects different interactions such as video transmission, display, user interactions, and other events occurring during the streaming. Event listeners and message buses can be used to accomplish the same.

The QoS and QoE Servers play an important role in the measurement and monitoring of the various network and user experience parameters to make sure that the smart contract terms based on the various SLAs and/or ELAs are adhered to by various participating entities. The Database stores all the required information of the network. It can be a centralized horizontally scalable (MongoDB) database or vertically scalable $(S Q L)$ or a decentralised data storage application such as Filecoin or SWARM.

\section{Technical Challenges and Open Issues}

1) Design of Scalable Blockchain-Based Video Streaming Platform: Video streaming in general consists of a large number of "sessions", which result in multiple transactions during a single stream. This may lead to a remarkably high number of transactions, which can lead to long delays and/or high computational complexity. Therefore, a development platform is critically required to implement the innovative strategies in a custom blockchain platform/model for video streaming to overcome the existing challenges of longer transaction times and limited computing power. The current efforts in this direction, for instance, from Play2Live, which used the Graphene platform - a $3^{\text {rd }}$ generation software with cryptographic capabilities capable of performing 50000 transactions per second, can be explored further.

2) Payments and Revenue Sharing Model: An exciting and much-required research challenge is the design of revenue models which are fair and maximize the benefits of all the involved stakeholders, e.g., content producer, publisher, aggregator, technology provider, advertiser, and legal services. At present, artist contracts and payments are severely limited due to the presence of such intermediaries. Blockchain with a smart contract can help to design an automated payment system, which ultimately allows artists and creators to get their rightful shares and allows them to retain ownership of their content. Along with the revenue model, the design of a proper Service Level Agreements (SLA) and Experience Level Agreements (ELA) is also required in order to assign tasks and responsibilities to each stakeholder.

3) Protection of Content Owners' Rights: A thorough investigation is required to develop the specific provision and features to help track the ownership and prevent illegal copying and distribution of the content. Applicationspecific smart contracts incorporating licensing and ownership related features will need to be developed, which possibly can help address the DRM issues, which remains an enormous challenge for the media industry.

4) Lack of Standards and Regulation: Currently, one of the most significant disadvantages for blockchain technology is the uncertainty about the legal status of its applications, such as the cryptocurrency, betting, and gambling. For the technology to mature and get accepted by different public and private entities, we need an international blockchain-based video streaming standardization group, including both technical and legal experts representing the industry, academia, and regulators. There is a need to develop video streaming related smart contract standards on the likes of ERC-20 Ethereum Smart Contract. Other standards to maintain interoperability between legacy and newly developed blockchain-based video streaming platforms such as Interledger Protocol also needs to be developed.

5) Integration and Interoperability: In order to make sure of interoperability with legacy systems, more research towards development of standard protocols and terminology is required. Also, new network technologies such as Fog computing, MEC, Software Defined Networking (SDN), and Network Functions Virtualization (NFV) are being introduced. Therefore, the designed solution should also be interoperable with newly developed technologies and protocols. Also, with the rise of multiple decentralized streaming companies, there is a need for standardization across various ledgers and tokens to ensure interoperability with different currencies/tokens on the blockchain.

6) Big Data Analytics for ML/AI: The data and information stored by the blockchain application can be used for data analytics. Blockchain technology with inherent data security features can provide additional functionality of automated tasks such as generating reports based on different ML and Artificial Intelligence (AI) algorithms. The computation can be performed more efficiently due to the availability of the joint computational power of the participating nodes. More efforts in this direction can help us understand and improve network efficiency and user satisfaction.

7) QoE Measurement, Modeling, and Control: QoE remains an integral part of today's video streaming applications. Since a blockchain-based video streaming platform is 
different from a traditional "centralized", video streaming platform as used currently, the applicability and efficiency of currently developed models for these newer applications remain an open question. Therefore, it is required to identify various influencing factors, $\mathrm{QoE}$ monitoring and control models, amongst others, designed for such applications. Furthermore, identifying such factors is required for successful enforcing of SLAs and ELAs between various stakeholders.

\section{Conclusion}

In this paper, we discussed the blockchain technology and its potential role and advantages for video streaming applications. We also investigated various existing applications that already implemented blockchain in video streaming and pointed out that there exists a significant gap between the industry and academia. Standardization will be an important enabler for the adoption of blockchain for media streaming. To address this aspect, we presented a discussion on the relevant activities in various standardization groups. We also presented a conceptual framework highlighting the possible network architecture and interaction between various elements. Based on our work, we identified key technical challenges and open research questions, which will help to bring the technology to the marketplace successfully.

\section{ACKNOWLEDGMENTS}

The authors acknowledge the support of the European Commission project H2020-643072 QoE-NET.

\section{RefERENCES}

[1] "TV streaming services overtake pay TV for first time." https://www.ofcom.org.uk/about-ofcom/latest/media/ media-releases/2018/streaming-overtakes-pay-tv, July 2018. [Online: Accessed 2-Nov-2019].

[2] N. Barman, An objective and subjective quality assessment for passive gaming video streaming. PhD thesis, Kingston University London, 2019.

[3] "Blockchain Market Shares, Market Strategies, and Market Forecasts, 2018 to 2024." https://www.ibm.com/downloads/cas/ PPRR983X, Jan 2018. [Online: Accessed 2-March-2020].

[4] "The Reality of Blockchain." https://www.gartner.com/ smarterwithgartner/the-reality-of-blockchain/, Oct 2018. [Online: Accessed 18-March-2020].

[5] M. S. Ali, M. Vecchio, M. Pincheira, K. Dolui, F. Antonelli, and M. H. Rehmani, "Applications of Blockchains in the Internet of Things: A Comprehensive Survey," IEEE Communications Surveys Tutorials, vol. 21, pp. 1676-1717, Secondquarter 2019.

[6] J. Qiu, D. Grace, G. Ding, J. Yao, and Q. Wu, "Blockchain-Based Secure Spectrum Trading for Unmanned-Aerial-Vehicle-Assisted Cellular Networks: An Operators Perspective," IEEE Internet of Things Journal, vol. 7, pp. 451-466, Jan 2020.

[7] Y. Jeong, D. Hwang, and K. Kim, "Blockchain-Based Management of Video Surveillance Systems," in 2019 International Conference on Information Networking (ICOIN), pp. 465-468, Jan 2019.

[8] S. S. Arslan and T. Goker, "Compress-Store on Blockchain: A Decentralized Data Processing and Immutable Storage for Multimedia Streaming," arXiv, vol. 1905.10458, May 2019.

[9] "Enforcing accountability in media - How blockchain technology can work for media and entertainment." https://www.ibm. com/downloads/cas/6146Z4JE, Sept 2018. [Online: Accessed 18March-2020].

[10] "Blockchain for Video Advertising: A Market Snapshot of Publisher and Buyer Use Cases." https://www.iab. com/wp-content/uploads/2018/02/Blockchain_for_Video_ Advertising_Publisher-Buyer_Use_Cases_2018-02.pdf, Feb 2018. [Online: Accessed 7-March-2020].
[11] "JPEG White paper: Towards a Standardized Framework for Media Blockchain and Distributed Ledger Technologies." https://jpeg.org/static/whitepapers/ jpeg-media-blockchain-whitepaper.pdf, August 2019. [Online: Accessed 22-March-2020].

[12] D. Bhowmik and T. Feng, "The multimedia blockchain: A distributed and tamper-proof media transaction framework," in 2017 22nd International Conference on Digital Signal Processing (DSP), pp. 1-5, Aug 2017.

[13] M. Zhaofeng, H. Weihua, and G. Hongmin, "A new blockchainbased trusted DRM scheme for built-in content protection," EURASIP Journal on Image and Video Processing, vol. 91, pp. 1116911185, Sept 2018.

[14] T. Bui, D. Cooper, J. Collomosse, M. Bell, A. Green, J. Sheridan, J. Higgins, A. Das, J. R. Keller, and O. Thereaux, "Tamperproofing Video with Hierarchical Attention Autoencoder Hashing on Blockchain," IEEE Transactions on Multimedia, pp. 1-1, 2020. Early Access.

[15] S. Ghimire, J. Y. Choi, and B. Lee, "Using blockchain for improved video integrity verification," IEEE Transactions on Multimedia, vol. 22, pp. 108-121, Jan 2020.

[16] H. R. Hasan and K. Salah, "Combating Deepfake Videos Using Blockchain and Smart Contracts," IEEE Access, vol. 7, pp. 4159641606, 2019.

[17] "JPEG Committee releases a call for evidence for image compression based on AI." https://jpeg.org/items/20200217_press.html, Feb 2020. [Online: Accessed 18-March-2020].

[18] N. Herbaut and N. Negru, "A Model for Collaborative BlockchainBased Video Delivery Relying on Advanced Network Services Chains," IEEE Communications Magazine, vol. 55, pp. 70-76, Sep. 2017.

[19] Y. Liu, F. R. Yu, X. Li, H. Ji, and V. C. M. Leung, “Decentralized Resource Allocation for Video Transcoding and Delivery in Blockchain-Based System With Mobile Edge Computing," IEEE Transactions on Vehicular Technology, vol. 68, pp. 11169-11185, Nov 2019.

[20] M. Liu, F. R. Yu, Y. Teng, V. C. M. Leung, and M. Song, “Distributed Resource Allocation in Blockchain-Based Video Streaming Systems With Mobile Edge Computing," IEEE Transactions on Wireless Communications, vol. 18, pp. 695-708, Jan 2019.

[21] Y. Liu, F. R. Yu, X. Li, H. Ji, and V. C. M. Leung, "Decentralized Resource Allocation for Video Transcoding and Delivery in Blockchain-Based System With Mobile Edge Computing," IEEE Transactions on Vehicular Technology, vol. 68, pp. 11169-11185, Nov 2019.

[22] "Twitch Affiliate Agreement." https://www.twitch.tv/p/en-gb/ legal/affiliate-agreement/, Aug 2018. [Online: Accessed 13March-2020].

[23] "Uber - Payments and Earnings." https://www.uber.com/ en-GH/drive/resources/payments/, 2019. [Online: Accessed 17March-2020]

[24] "Revealed: 50 million Facebook profiles harvested for Cambridge Analytica in major data breach." https://www.theguardian.com/news/2018/mar/17/ cambridge-analytica-facebook-influence-us-election, Mar 2018. [Online: Accessed 17-March-2020].

[25] "Flixxo - Based on a true story." https://medium.com/@flixxo/ based-on-a-true-story-12e8e8b51bc0, Sept 2019. [Online: Accessed 7-March-2020]

[26] "VideoCoin Analysis." https://medium.com/dcryptinc/ videocoin-analysis-a3d433b9a126, Oct 2018. [Online: Accessed 13-March-2020].

[27] D. Puthal, N. Malik, S. P. Mohanty, E. Kougianos, and G. Das, "Everything You Wanted to Know About the Blockchain: Its Promise, Components, Processes, and Problems," IEEE Consumer Electronics Magazine, vol. 7, pp. 6-14, July 2018.

[28] T. Aste, P. Tasca, and T. Di Matteo, "Blockchain Technologies: The Foreseeable Impact on Society and Industry," Computer, vol. 50, pp. 18-28, Sept 2017. 
Nabajeet Barman [S'16, M'19] received PhD in 2019 from Kingston University, MSc in Information Technology from Universität Stuttgart, Germany, and B.Tech in Electronics Engineering from NIT Surat, India. He was an MSCA Fellow, where he worked on the H2020 EU QoENet project on Quality of Experience aware video coding strategies. Currently Research Associate at Kingston University, he is a Video Quality Expert Group (VQEG) Board member and is also involved in ITU$T$ standardization activities. His research interests include multimedia communications and machine learning and more recently distributed ledger technologies.

Deepak G C [S'12, M'16] received $\mathrm{PhD}$ in 2017 from Lancaster University, InfoLab21, UK, MSc in Computer Engineering from Chonbuk National University, South Korea, and BEng degree in Electronics Engineering from Pokhara University, Nepal. He is a Lecturer at the School of Computer Science and Mathematics, Kingston University London. He has previously worked as a research fellow at Liverpool John Moores University, U.K., under EU H2020 project Wi-5 and Kingston University London under EPSRC GCRF Project DARE. His research interest includes Radio Access Technologies, Cognitive Radio, 5G, Physical Layer Security, Internet-of-Things, Cyber Security, and Public Safety Communications.

Maria Martini [SrM'07] is Professor in the Faculty of Science, Engineering and Computing at Kingston University, London, U.K., where she also leads the Wireless Multimedia Networking Research Group and she is the Course Director for the MSc in "Networking and Data Communications." She is a Fellow of The Higher Education Academy (HEA). She has led the KU team in a number of national and international research projects, funded by the European Commission (e.g., OPTIMIX, CONCERTO, QoE-NET, Qualinet), U.K. research councils, U.K. Technology Strategy Board/InnovateUK, and international industries. She received the Laurea degree in electronic engineering (summa cum laude) from the University of Perugia, Italy, in 1998 and the PhD degree in Electronics and Computer Science from the University of Bologna, Italy, in 2002. 